\section{Long-term data at Research and Extension Centers to fuel new informatics program}

U nderstanding and managing the myriad environments that drive our natural world requires science that can illuminate the systems in play and how they interact, across time and spatial scales. There are efforts across UC and beyond to bring together such information, transforming our understanding of global change and enabling scientists to make predictions about how ecosystems will respond.

Such efforts use informatics, the convergence of biology, geography and computer science. The collection, analysis and visualization of complex data are now critical in research, business and government. Increasingly, we can uncover the meanings behind interrelated data sets and use them to make projections. But the models are only as good as the data. We need rich and extensive data, gathered from and usable across disciplines - whether by ecologists, geographers, hydrologists, managers, modelers, farmers or others.

Through a new statewide program, UC Agriculture and Natural Resources (ANR) will organize, digitize and make Web-accessible some of California's longest continuous data on agriculture and natural ecosystems, including weather and productivity related to management inputs - concrete data for modeling responses to change across the state.

Led by UC Cooperative Extension Specialist Maggi Kelly, housed at UC Berkeley, and Lisa Fischer, associate director of ANR's Research and Extension Center system, the new Informatics and Geographic Information Systems statewide program (IGIS) is now constructing databases and methodologies to ensure wide Web accessibility to decades of findings.

For the last 50 to 100 years, data has been carefully collected and recorded at nine RECs across the state. The centers span California from the Oregon border to Mexico, and represent a wide variety of geographic, topographic and climatic zones. Each includes land and facilities. There are two large (5,000-plus-acre) oak woodland-dominated sites with livestock grazing, and seven smaller (140- to 330-acre) sites dominated by intensively managed agricultural crops using a variety of cropping systems.

Over the years, baseline data collection (e.g., weather, soils and more) and research projects have generated a large body of findings. However, most of it is unavailable outside individual centers, and in many cases unavailable beyond the research team. This lessens the value of the projects, and by extension the RECs, to the larger academic and management world.

The new project will make REC research data available to scientists, managers and the public interested in understanding and modeling our complex world. This will enable scientists to make meaningful predictions of the agricultural and ecosystem responses to change, enhancing agricultural productivity in the future. Models alone do not yet provide an adequate basis for decisions about which crops are likely or decrease, where to do well or poorly, whether rangeland and forest productivity will increase

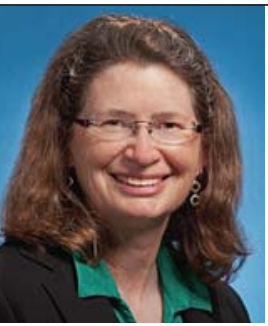

Barbara Allen-Diaz

Vice President

UC Agriculture and Natural Resources

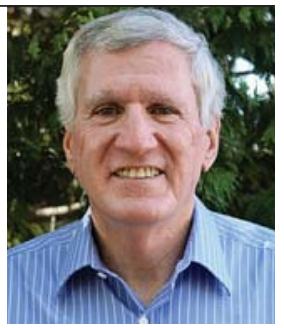

Bill Frost

Associate Director Cooperative Extension and the Agricultural Experiment Station

UC Agriculture and Natural Resources amounts and patterns are likely to change or where biodiversity shifts are likely to occur. But models gain predictive power through sufficient data, and the RECs' storehouse of data can make a strong contribution.

In addition, models based on multiple years of data can project trends, predict thresholds for agricultural land conservation efforts, inform policy to conserve prime agricultural land and help meet the demand for food, fiber, fuel and ecosystem services under changing economic, environmental and demographic conditions.

Complex data sets must be acquired from paper records at the RECs and from researchers willing to share their original findings, and then digitized, stored and made searchable, discoverable and accessible. One example of baseline data is the historical record of the centers' weather, vegetation type, soil type, soil fertility, and soil chemical analysis, all specified according to mapped plots, pastures and boundaries.

Specific to individual centers, data will be gathered on numbers of grazing animals by pasture, leaf analyses for tree fertility, fruit quality data and specific disease or invasive species issues. Other findings include raw field data collected for specific research projects, summarized plot data, written reports and journal articles, including those in California Agriculture, ANR's premier vehicle for research dissemination. Lastly, new data will be compiled at each center through a new instrumentation system, which includes an eddy covariance system tower, meteorological equipment and a soil sensor network compatible with national ecosystem observation networks to provide air and soil data.

This issue of California Agriculture reports research demonstrating the value of UC data collections: a survey of how Williamson Act cuts could affect private ranch land (page 131); an analysis of water use by oak woodlands and vineyards in Sonoma County (page 144); a review of how the United Nations' Clean Development Mechanism could help California achieve greenhouse gas reduction goals (page 137); a study of job mobility among dairy workers (page 153) and an evaluation of a new $4-\mathrm{H}$ water education curriculum to improve science literacy (page 158).

The IGIS project will soon invite UC scientists to contribute their REC research data and include them in conversations about database construction and methodologies. Providing access to the wealth and breadth of data available from the UC ANR REC system will serve researchers, managers and the public for generations to come. 